\title{
Collaborative Recommendation of Tourist Attractions Based on the Comprehensive Similarity of Users
}

\author{
Jinlong Chen ${ }^{1}$, JiaLing $\mathrm{Li}^{2}+$ \\ ${ }^{1}$ Guangxi Key Laboratory of Cryptography and Information Security , Guilin University of Electronic \\ Technology, China \\ ${ }^{2}$ Guangxi Key Laboratory of Trusted Software, Guilin University of Electronic Technology, China
}

\begin{abstract}
One problem in collaborative filtering recommendation is the sparseness of the rating data, which affects the quality of system recommendations. We propose a new method which uses the similarity of the image formed by the user's personality tags, the similarity of the user's historical rating records, and the similarity of the attention among friends to make up for the lack of calculating similarity using only rating data. The three similarities are multiplied by their respective weights and combined to obtain a comprehensive similarity for prediction. The experimental results show that the improved similarity method can better distinguish the similarity between users and users, effectively improve the accuracy of neighbor selection, reduce the average absolute error between the predicted score and the actual score, and improve the accuracy of the score prediction.
\end{abstract}

Keywords: Rating prediction, collaborative filtering, user personality tag, Friend attention, recommendati on algorithm, similarity.

\section{Introduction}

With the rocket-like progress of the Internet, for example online shopping, social networking, instant messaging, online listening to music, and watching movies, make our daily lives more colorful. People deal with huge amounts of data every day. How to let people quickly find the information they need and obtain useful data in massive data? Recommendation systems have been generated from this, Currently, these systems are incorporating social information [1]. It recommends its users according to their needs. Required information. For example, in the field of e-commerce, according to the user's historical behavior (click, browse, favorite, add to cart, purchase, etc.) to capture the user's interests, and then recommend products that meet his preferences to the user. It saves the user's search time for searching information; On the other hand, it can accurately recommend items to users, bringing certain economic benefits to the industry. Mobile phone travel software recommends and recommends attractions to become the product of Internet development. At present, the personalized recommendation system, which is based on the product's historical score, plays an important role in predicting customer needs and making users interested in specific products[2]. There are currently three types of recommendation systems: content filtering -based filtering (CBF) model, collaborative filtering (CF) model and hybrid models (HM). Collaborative filtering is a popular recommendation technique[3]. A large number of examples can prove that it is a very successful algorithm in the recommendation algorithm [4]. In the collaborative filtering recommender system, the function of the system is to select the likely users' favorite items recommended to them based on their interests[5].Collaborative filtering recommendation algorithms can be subdivided into user-based collaborative filtering algorithms and item-based collaborative filtering algorithms. The working process of the collaborative filtering recommendation algorithm can be described as: using the data generated by the

\footnotetext{
+ Corresponding author. Tel.: Tel:+86 15677388659..

E-mail address: 2602481415.
} 
user's historical behavior to calculate the similarity between two users or similarity between the purchase and other purchase, and then in descending order of similarity Sort them in order, and then take the top-N users with the highest similarity as their neighbor users to make recommendations for users. Therefore, the key to this algorithm is to use the user's purchase rating matrix to find similar App customers or goods in order to the system can be able to recommend the right product to the user[6].

However, due to the direct result of sparse scoring, the recommender algorithm may provide poor recommendations or reject recommendations[7]. the author proposes an improved method. The first is to use the user's personality tags to construct the user's image similarity, then calculate the friend's attention similarity between the users based on the friends whom the users follows, and finally calculate the user-touser preference similarity based on the user's common score. The similarity is multiplied by the weights and combined to obtain the final comprehensive similarity. The top- $\mathrm{N}$ users with the highest similarity are selected as the user's neighbor user set, then this user's rating of the certain items is predicted, finally recommend scenic spots with high score for this user .

\section{Traditional Scoring Prediction Method}

The traditional scoring and forecasting method is usually based on the user's historical scoring data, by analyzing preference and hobbies of this user, using the calculation method of Pearson correlation coefficient to select out the users with similar interests and hobbies to this user in the user group, find the top-N users with the highest similarity of interests and hobbies, and then evaluate some items according to these users to predict the target user's interest to the scenic spots. It is usually divided into two steps: 1. Similarity calculation, 2. Scoring prediction.

\subsection{Similarity Calculation}

It is assumed that a set of $m$ users is represented by $U(U n$ represents the Nth user), and a set of $n$ scenic spots is represented by I(In represents the Nth scenic spot). Then the user rating data set is written as an $\mathrm{m} \times \mathrm{n}$ matrix, as shown in Table 1.

Table 1: Scoring matrix R ( $\mathrm{mX} \mathrm{n})$

\begin{tabular}{|c|c|c|c|c|}
\hline & I1 & 12 & $\ldots$ & In \\
\hline $\mathbf{U}_{1}$ & ${ }^{\mathrm{R}} \mathrm{u} 1,1$ & ${ }^{\mathrm{R}} \mathrm{u} 1,2$ & $\ldots$ & ${ }^{\mathrm{R}} \mathbf{u} 1, \mathbf{n}$ \\
\hline $\mathbf{U}_{2}$ & ${ }^{\mathrm{R}} \mathrm{u} 2,1$ & ${ }^{\mathrm{R}} \mathrm{u} 2 ， 2$ & $\ldots$ & ${ }^{R} \mathbf{u} 2, n$ \\
\hline$\ldots$ & $\ldots$ & $\ldots$ & $\ldots$ & $\ldots$ \\
\hline $\mathbf{U}_{\mathbf{m}}$ & ${ }^{\mathrm{R}} \mathrm{um}, 1$ & ${ }^{\mathrm{R}} \mathrm{um}, 2$ & $\ldots$ & ${ }^{\mathrm{R}} \mathrm{um}, \mathrm{n}$ \\
\hline
\end{tabular}

Suppose a user $U_{k}(k \in m)$ scores $R_{u k, j}$ for scenic spots $I_{j}(j \in n)$. The score represents the target user's interest degree to scenic spots. In the scoring system, the similarity between users is usually calculated by using the Pearson correlation coefficient. The calculation formula is as follows.

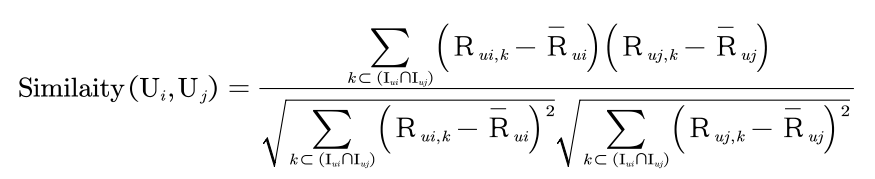

Among them, $\mathrm{U}_{i}$ represents user $\mathrm{i}, \mathrm{U}_{j}$ represents user $\mathrm{j}, \mathrm{I}_{u i} \cap \mathrm{I}_{u j}$ represents the scenic spot that user $\mathrm{i}$ and user $\mathrm{j}$ have both visited, Similaity $\left(\mathrm{U}_{i}, \mathrm{U}_{j}\right)$ is the similarity score of user $\mathrm{i}$ and user $\mathrm{j}, \mathrm{R}_{u i, k}$ represents the score of the user $\mathrm{i}$ on scenic spot $\mathrm{k}$, and $\mathrm{R}_{u i}$ represents the average of user i score, $\mathrm{R}_{u j, k}$ represents user $\mathrm{j}$ 's rating of scenic area $k$, and $\overline{\mathrm{R}}_{u j}$ mean user $\mathrm{j}$ 's average rating.

\subsection{Score Prediction}

Secondly, similarity between users is sorted in descending order, and then select the Top-N users with the highest similarity and they has been the user's neighbors, and the target is predicted based on the 
historical scores of neighbors on a scenic spot Users will rate this scenic spot. The prediction formula [8]is as follows.

$$
\hat{\mathrm{R}}_{u i, k}=\overline{\mathrm{R}}_{u i}+\frac{\sum_{j=1, \mathrm{U}_{j} \subset \mathrm{U}_{\mathrm{N}}}^{\mathrm{N}} \operatorname{Similaity}\left(\mathrm{U}_{i}, \mathrm{U}_{j}\right)\left(\mathrm{R}_{u i, k}-\overline{\mathrm{R}}_{u}\right)}{\sum_{j=1, \mathrm{U}_{j} \subset \mathrm{U}_{\mathrm{N}}}^{\mathrm{N}} \operatorname{Similaity}\left(\mathrm{U}_{i}, \mathrm{U}_{j}\right)}
$$

Among them, $\mathrm{U}_{N}$ represents the neighbor set of this target user, Similaity $\left(\mathrm{U}_{i}, \mathrm{U}_{j}\right)$ is expressed as the similarity between user $\mathrm{i}$ and user $\mathrm{j}$, and $\hat{\mathrm{R}}_{u i, k}$ represents this target i's prediction score for scenic area $\mathrm{k}$.

\section{Scoring Prediction Algorithm Based on User Comprehensive Similarity}

The traditional rating prediction method is to depend on the similarity between users by the user's historical rating to the scenic spots. In actual life, this situation does not necessarily happen when two users have browsed the same scenic spot. For example, users a and b have visited the same scenic spot, watched the same movie, heard the same song, or bought the same Goods, etc. But some users are unwilling to spend time for scenic spot ratings, so The traditional collaborative filtering model has high-dimensional sparse user evaluation information and ignores the user preference information contained in user evaluation[9] In view of the above problems, the author proposes a rating prediction recommendation algorithm by the comprehensive similarity of users. The working principle of the algorithm: This method calculates the similarity of user images, the similarity of user historical rating records, and the degree of attention among friends. Similarity, which makes up for the lack of calculating similarity using only scoring data. Through the similarity of three indicators, the three similarities are multiplied by their respective weights to combine to obtain a comprehensive similarity for prediction.

\subsection{Calculation of Comprehensive Similarity Index Values}

- Image Similarity Index

When the user signs up for an account, the website will give some user-friendly image characteristics to choose from, such as " Climbing fans", " food aficionado" and other personal tags, shaping the user's personal image, for a example, $\overrightarrow{\mathrm{U}}_{1}=\left[\mathrm{P}_{11}, \mathrm{P}_{12}, \mathrm{P}_{13}, \ldots, \mathrm{P}_{1 s}\right]$,Among them, $\mathrm{P}_{11}$ represents the first image characteristics of user 1 , and $P_{1 s}$ represents the $s$ image characteristic of user 1 . After the user's personality image is determined, the similarity of personality tags between users is expressed as follows:

$$
\operatorname{Similaity}\left(\overrightarrow{\mathrm{U}}_{i}, \overrightarrow{\mathrm{U}}_{j}\right)=\overrightarrow{\mathrm{U}}_{i} \cdot \overrightarrow{\mathrm{U}}_{j}=\frac{\sum_{a=1}^{\mathrm{s}}\left(\mathrm{P}_{i a} \times \mathrm{P}_{j a}\right)}{\sqrt{\sum_{i=1}^{\mathrm{s}} \mathrm{P}^{2}{ }_{i a}} \sqrt{\sum_{i=1}^{\mathrm{s}} \mathrm{P}^{2}{ }_{j a}}}
$$

Where $\overrightarrow{\mathrm{U}}_{i}$ represents the personality tag vector of user $\mathrm{i}, \overrightarrow{\mathrm{U}}_{j}$ represents the personality tag vector of user $\mathrm{j}$, and Similaity $\left(\overrightarrow{\mathrm{U}}_{i}, \overrightarrow{\mathrm{U}}_{j}\right)$ is the image similarity between $\mathrm{i}$ and $\mathrm{j}$.

- Friends attention index

The attention between users and the social relationship information being followed play a key role in the recommendation. The value of the friend attention index between two users is calculated as Equation (4):

$$
F\left(U_{\mathrm{i}}, U_{\mathrm{j}}\right)=\frac{\mathrm{M}_{U_{\mathrm{i}} \cap U_{\mathrm{j}}}}{\max _{1 \leqslant n \ll s}\left(U_{\mathrm{i}} \cap U_{n}\right)}
$$

In the formula, it indicates the friend attention index between user $U_{i}$ and other users, s means $U_{i}$ 's all the 
friends, $\mathrm{M}_{U_{\mathrm{i}} \cap U_{\mathrm{j}}}$ is the number of mutual friends followed by $\mathrm{U}_{\mathrm{i}}$ and $\mathrm{U}_{\mathrm{j}}$, and $\max _{1 \leqslant n \leqslant s}\left(\mathrm{U}_{\mathrm{i}} \cap U_{n}\right)$ is the maximum value of the number of friends in common between the user $u$ and all the friends he follows.

- Preference Similarity Index

The closer a user scores to the same item, the more similar the preferences between users. By calculating the average of the score differences and normalizing them, the preference similarity index values between users are obtained, as shown in equation (5):

$$
\operatorname{Similaity}\left(\mathrm{U}_{\mathrm{i}}, \mathrm{U}_{\mathrm{j}}\right)=\frac{\sum_{\mathrm{k} \subset\left(\mathrm{I}_{\mathrm{u}} \cap \mathrm{N}_{\mathrm{w}}\right)}\left(\mathrm{R}_{\mathrm{ui}, \mathrm{k}}-\overline{\mathrm{R}}_{\mathrm{ui}}\right)\left(\mathrm{R}_{\mathrm{uj}, \mathrm{k}}-\overline{\mathrm{R}}_{\mathrm{uj}}\right)}{\sqrt{\sum_{\mathrm{k} \subset\left(\mathrm{I}_{\mathrm{u}} \cap \mathrm{I}_{\mathrm{w}}\right)}\left(\mathrm{R}_{\mathrm{u}, \mathrm{k}, \mathrm{k}}-\overline{\mathrm{R}}_{\mathrm{ui}}\right)^{2}} \sqrt{\sum_{\mathrm{k} \subset\left(\mathrm{I}_{\mathrm{u}} \cap_{\mathrm{i}} \mathrm{u}\right)}\left(\mathrm{R}_{\mathrm{uj}, \mathrm{k}}-\overline{\mathrm{R}}_{\mathrm{uj}}\right)^{2}}}
$$

Among them, $U_{i}$ represents user $i, U_{j}$ represents user $j, I_{u i} \cap I_{u j}$ represents scenic spots that user $i$ and user $\mathrm{j}$ have both viewed, Similaity $\left(\mathrm{U}_{i}, \mathrm{U}_{j}\right)$ representing the similarity of preferences of $\mathrm{i}$ and $\mathrm{j}$ to the visited scenic spots, and $\mathrm{R}_{\mathrm{ui}}, \mathrm{k}$ represents the rating of scenic area $\mathrm{k}$ of $\mathrm{i}, \mathrm{R}_{u i}$ represents the average rating of i. $R_{u j, k}$ represents $\mathrm{j}$ 's rating of scenic area $\mathrm{k}$, and $\overline{\mathrm{R}}_{u j}$ mean user $\mathrm{j}$ 's average rating.

\subsection{Comprehensive Similarity Calculation}

For users, the larger the number of the comprehensive similarity index, the more similar the users are. The user's image similarity index, friend attention index, and preference similarity index are integrated, The comprehensive similarity of $i$ and $j$ is shown in (6):

$$
\operatorname{sim}(i, j)=w_{1} \bullet \operatorname{Similaity}\left(\overrightarrow{\mathrm{U}}_{i}, \overrightarrow{\mathrm{U}}_{j}\right)+w_{2} \bullet F\left(U_{\mathrm{i}}, U_{\mathrm{j}}\right)+w_{3} \bullet \operatorname{Similaity}\left(\mathrm{U}_{i}, \mathrm{U}_{j}\right)
$$

Where $w_{1}, w_{2}, w_{3}$ represents the weight of each part, $w_{1}+w_{2}+w_{3}=1$.

\subsection{Nearest Neighbor Dataset Selection}

In the field of recommendation systems, the nearest neighbor-based method is one of the earliest methods. Different users will have different recommendation effects. According to the neighbor dataset established for each user, $\mathrm{k}$ neighbor users are selected as the user's neighbor dataset .

\subsection{Combine Similarities and Predict Scores}

After obtaining the neighbor data set, formula (2) is used so that the system can calculate the user's prediction scores to the scenic spots.

\section{Experiment.}

\subsection{Experimental Data Set}

- Data collection

The article uses crawler technology to get the data of travel websites, and obtain the required experimental data through content analysis. Information on tourist attractions in Xiamen, Lijiang, Hangzhou, Guilin and other cities was collected through travel websites, as well as user evaluations of these attractions, user-friendship relationships, and user image type tags. The user rating set is obtained by selecting 30090 pieces of rating data from 670 users on 854 scenic spots in the travel data set; the image tag data set collected 236 user image type tags and 5,100 image data of 670 users; the user attention data set was collected The friend relationship between users constitutes a friend matrix. If user $\mathrm{i}$ and $\mathrm{j}$ have concerns, they are marked as friends, and the value in the row $i$ and the column $j$ of the matrix are assigned a value of 1 and 0 otherwise.

- data preprocessing

In order to make the experimental results more accurate, the experimental data must be processed:

(1) Delete the user's rating of duplicate scenic spots, then select the user's nearest rating for their scenic spots as experimental data;

(2) Delete attractions without user ratings.

After processing, the user rating set becomes 29720 rating data from 670 users to 840 scenic spots in the travel data set. 


\subsection{Evaluation Criteria}

Accuracy is one of the most basic indicators in evaluating a recommendation system. In this paper, the average absolute error MAE and the root mean square error RMSE are used as accuracy metrics [10].

The smaller the average absolute error MAE, the more accurate the recommendation. Suppose the set of user's predicted ratings is $\{\mathrm{p} 1, \mathrm{p} 2, \ldots, \mathrm{pn}\}$, and the corresponding user's actual rating set is $\{\mathrm{q} 1, \mathrm{q} 2, \ldots, \mathrm{qn}\}$, where $\mathrm{n}$ represents the number of all predicted rating products, then MAE's the calculation formula is shown in (7):

$$
\mathrm{MAE}=\frac{\sum_{\mathrm{i}=1}^{\mathrm{n}}\left|p_{\mathrm{i}}-q_{\mathrm{i}}\right|}{\mathrm{n}}
$$

The smaller the value of RMSE, the higher the recommended quality. the formula for calculating RMSE (8):

$$
\mathrm{RMSE}=\sqrt{\frac{\sum_{\mathrm{i}=1}^{\mathrm{n}}\left(\mathrm{p}_{\mathrm{i}}-\mathrm{q}_{\mathrm{i}}\right)^{2}}{\mathrm{n}}}
$$

\subsection{Comparative Experimental Eesign and Results Analysis}

The algorithm adopted in the experiment is compared as follows:

1. UBCF. The user-based collaborative filtering recommendation algorithm in [11].

2. TrustCF. (Trust-aware Recommendation). A social recommendation algorithm for collaborative filtering recommendation based on trust consciousness proposed by [12].

3. CoCF (Coordination filtering based on user comprehensive similarity). This paper proposes a collaborative filtering algorithm based on user comprehensive similarity.

In this paper, experiments are performed on the UBCF algorithm, TrustCF algorithm, and CoCF algorithm, and the accuracy of the algorithm is evaluated by calculating the MAE and RMSE of the experimental results. To improve the authenticity of the simulation results, the score set in the data set is randomly divided into two parts according to the ratio of 8: 2, which are the training set and the test set. In the formula of comprehensive similarity, we use the enumeration method to change the weight value to observe the changes in MAE and RMSE, and finally found that when $w 1=0.3$, w2 $=0.4$, w3 $=0.3$, the accuracy is the highest.

(1) Comparative analysis of MAE and RMSE under different algorithms

The DGECF algorithm selects the nearest neighbor values $(10,20,22,24,26,28,40,50,60,70)$ for prediction score, and calculates MAE and RMSE. The experimental results are shown in Figure 1 and Figure 2.

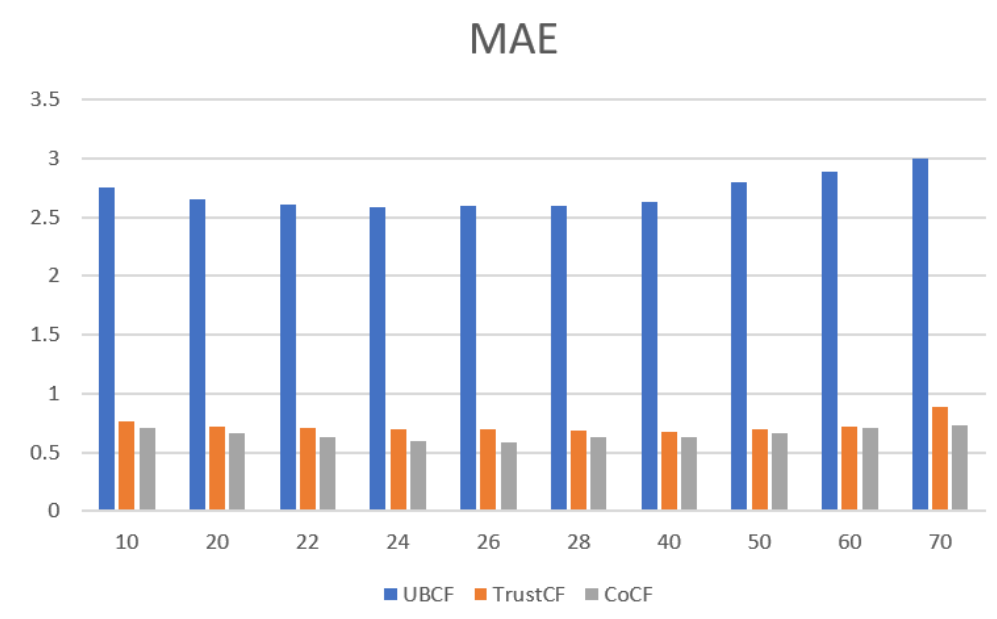

Fig 1: MAE histogram of three algorithms 


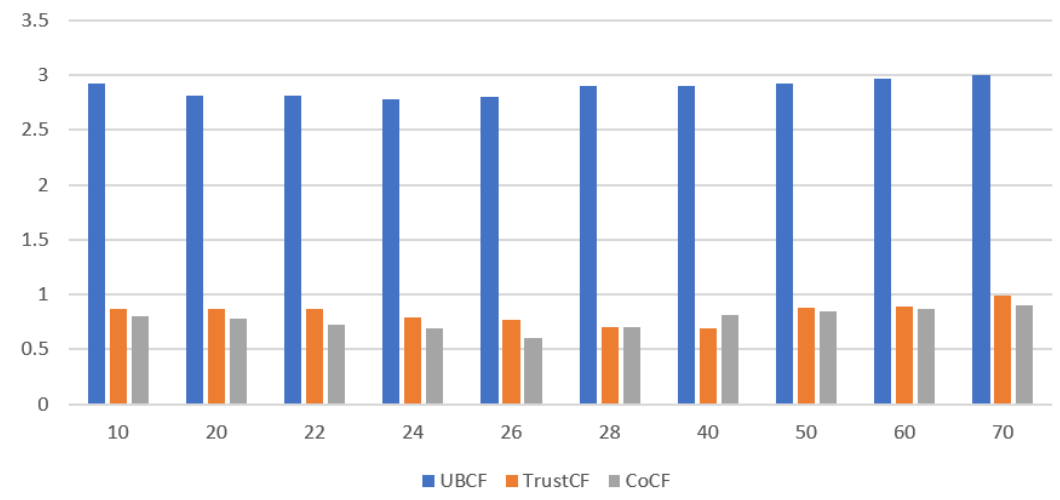

Fig 2: RMSE histogram of three algorithms

It can be seen from Figures 1 and 2 that in the UBCF algorithm, as the neighbor value $\mathrm{k}$ increases, MAE and RMSE both decrease and then rise. When the neighbor value is 24, MAE and RMSE are the lowest, which are: 2.5813 and 2.7842; in TrustCF In the algorithm, MAE and RMSE both show a downward trend and then increase with increasing $\mathrm{k}$. When $\mathrm{k}$ is 40, MAE and RMSE are the lowest: 0.6721 and 0.6944, respectively. In the CoCF algorithm, MAE and RMSE both follow The increase of $\mathrm{k}$ decreases first and then increases slightly, and when the nearest neighbor value $\mathrm{k}$ is 26, the MAE and RMSE are the lowest: 0.5855 and 0.6059, respectively. When the number of Top-N increases, the MAE and RMSE values of the three algorithms Both show a downward trend and an upward trend. This is because with the increase of recommended scenic spots, some scenic spots that the target user does not like will also be recommended to the target user, and the accuracy will also decrease. In addition, the improved algorithm has always been lower than the MAE and RMSE of the other two algorithms, indicating that the recommendation algorithm that uses comprehensive similarity can help target users find areas of interest and increase user appeal.

(2) Comparison and analysis of the average values of MAE and RMSE under different algorithms

Table 2: The average values of MAE and RMSE of three algorithms

\begin{tabular}{|l|l|l|l|}
\hline & UBCF & $\begin{array}{l}\text { Trust } \\
\text { CF }\end{array}$ & CoCF \\
\hline MAE & 2.7101 & 0.7224 & 0.6520 \\
\hline RMSE & 2.8824 & 0.8311 & 0.7735 \\
\hline
\end{tabular}

It can be seen from Table 2 that the average values of MAE and RMSE of the CoCF algorithm proposed in this paper are the lowest: 0.6520 and 0.7735 , respectively. Compared with the UBCF algorithm and the TrustCF algorithm, MAE is reduced by $75.94 \%$ and $9.75 \%$, and RMSE is reduced by $73.16 \%$ And 6.93\%, indicating that the CoCF algorithm has higher accuracy.

\section{Summary}

The rating prediction recommendation algorithm which the paper presents is based on user comprehensive similarity, which is an improvement on the traditional user-based recommendation coordination filtering algorithm. The working principle is that this method compensates for the shortcomings of using the scoring data to calculate the similarity by calculating the similarity of the user's image, the similarity of the user's historical records of the scenic spots, and the similarity of attention among friends. The three similarities are multiplied by their respective weights and combined to obtain a comprehensive similarity for prediction. The feature of this article is that it can solve the sparsity of the user-scored scenic area matrix. Through analyzing the experimental results, we can see the improved propulsion algorithm has a smaller average absolute error and a higher prediction accuracy than the traditional user-based recommendation coordination filtering algorithm. 


\section{Acknowledgements}

This research work is supported by the grant of Guangxi science and technology development project (No: AB17195027), the grant of Guangxi scientific research and technology development plan project (No: AB18221011), the grant of Guangxi Key Laboratory of Trusted Software of Guilin University of Electronic Technology (No: KX201620), the grant of Guilin science and technology project Research and application demonstration of key technologies of somatosensory interaction based on gesture (No: 20180107-4).

\section{References}

[1] Authors’ background J. Bobadilla,F. Ortega,A. Hernando,A. Gutiérrez. Recommender systems survey[J]. Knowledge-Based Systems,2013,46.

[2] Velammal B.L..Typicality - based collaborative filtering for book recommendation[J].Expert Systems,2019,36(3).

[3] Deepa Anand,Kamal K. Bharadwaj. Utilizing various sparsity measures for enhancing accuracy of collaborative recommender systems based on local and global similarities[J]. Expert Systems With Applications,2011,38(5).

[4] Yewang Chen,Cheng Wang,Shiqi Wen, et al.User-based collaborative filtering algorithm fusing the local and global nearest neighbour[J].International Journal of Internet Manufacturing and Services,2018,5(2/3):260-278.

[5] Kaedi, Marjan,Hafshejani, Zahra Yusefi,Fatemi, Afsaneh.Improving sparsity and new user problems in collaborative filtering by clustering the personality factors[J].Electronic commerce research,2018,18(4):813-836.

[6] Haifeng Liu,Zheng Hu,Ahmad Mian,Hui Tian,Xuzhen Zhu. A new user similarity model to improve the accuracy of collaborative filtering[J]. Knowledge-Based Systems,2014,56.

[7] Manzato, Marcelo G.,Campello, Ricardo J. G. B.,da Costa, Arthur F..Boosting collaborative filtering with an ensemble of co-trained recommenders[J].Expert Systems with Application,2019,115(Jan.):427-441.

[8] Valcarce, Daniel,Barreiro, Alvaro,Parapar, Javier.Finding and analysing good neighbourhoods to improve collaborative filtering[J].Knowledge-based systems,2018,159(Nov.1):193-202

[9] Heyong Wang,Ming Hong,Jinjiong Lan.Study on Collaborative Filtering Recommendation Model Fusing User Reviews[J].Journal of Advanced Computational Intelligence and Intelligent Informatics,2019,23(5):864-873.

[10] Bushra Alhijawi,Ayoub Alsarhan,Yousef Kilani.Using artificial intelligence techniques in collaborative filtering recommender systems: survey[J].International journal of advanced intelligence paradigms,2018,11(3/4):378-396.

[11]Y.L.Zhang. Research of User-Based Collaborative Filtering Recommendation Algorithm Based on Hadoop[C]. Science and Engineering Research Center.Proceedings of 2015 International Conference on Computer Information Systems and Industrial Applications(CISIA2015).Science and Engineering Research Center:Science and Engineering Research Center,2015:86-89.

[12] MASSA P, AVESANI P. Trust-aware collaborative filtering for recommender systems [C] // Proceedings of the 2004 Conference on Cooperative Information Systems. Berlin: Springer,2004:492 - 508 . 